\title{
How Translators Affect the Experience and Healthcare of Primary Spanish-Speaking Patients in the Trauma Department
}

Catherin Morocho ${ }^{1}$, Tasha Sparks Joplin ${ }^{1}$, Kevin Lopez ${ }^{1}$, Damaris Ortiz ${ }^{1,2}$, Craig J. Goergen ${ }^{3}$, Ashley Meagher ${ }^{1,4}$

Indiana University School of Medicine ${ }^{1}$, Sidney \& Lois Eskenazi Hospital Department of Surgical Trauma/Critical Care and Acute Care ${ }^{2}$; Purdue University Weldon School of Biomedical Engineering ${ }^{3}$; IU Health Methodist Department of Surgical Trauma/Critical Care and Acute Care ${ }^{4}$

Background and Objective: The trauma bay is a fast-paced environment where comprehension of medical jargon is difficult even for native English-speaking patients. For Spanish-speaking patients, the presence, and use of the translating tools in hospitals may change the course and/or quality of their care, especially in a trauma setting. Our objective was to gather information and perspectives of Spanish-speaking patients in the trauma bay and subsequent hospitalization. This pilot study determined if there were constant themes.

Methods: In this pilot qualitative study, we successfully recruited three adult primary Spanishspeaking patients admitted to the trauma service for at least twenty-four hours in July 2021 at an urban academic level I trauma center. Spanish only in-person semi-structured interviews were used to gather patient's perspectives, with data supplemented from electronic health records and trauma registries. The interview was transcribed in Spanish, translated to English, coded, and analyzed using thematic analysis.

Results: Although recruitment occurred at both hospitals, this study includes only three patients admitted at IU Health Methodist. All three were males aged 22-37 years from Latin America. Blunt injuries occurred in two with injury severity scores (ISS) ranging from 5-11 while the third had penetrating injuries with an ISS of 10 . Several themes have emerged. All patients felt they did not have autonomy or empowerment in their care. It was found that the healthcare team decided who received a translator. Two of the patients had a lack of understanding in their traumatic injuries. One patient relied on his partner for translating, even though a translator was provided.

Conclusions: These results suggest that Spanish-speaking trauma patients lack autonomy, empowerment, and understanding their medical conditions. Further interviews need to be conducted in order to strengthen the perspective of a Spanish-speaking trauma patient's care. 